\title{
RESEÑA
}

\section{Estrategias económicas exitosas en Asia y en América Latina ${ }^{1}$}

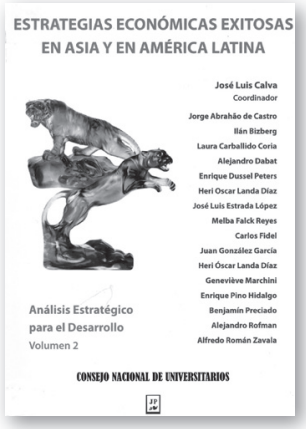

orientando sus tareas y actividades para la consecución de dicho objetivo. Así, durante los años sesenta se hablaba con optimismo de la "primera década del desarrollo".

Desde esos primeros años en que la idea y la búsqueda del desarrollo se colocaron en lo alto de la agenda internacional, las prácticas, actividades y estrategias aplicadas fueron de la mano, fundamentalmente, de las directrices marcadas por los países industrializados, bajo el supuesto de la existencia de un modelo de desarrollo único y aplicable a todos los países del mundo:

Santiago Aceves: Profesor del Instituto Tecnológico y de Estudios Superiores de Occidente (ITESO).

1. José Luis Calva, (coord.), México: Juan Pablos Editor/Consejo Nacional de Universitarios para una Nueva Estrategia de Desarrollo, 2012.

2. Concepto con el cual se refirió el presidente Harry S. Truman a los países en desarrollo durante su discurso inaugural en 1949, mismo que es visto por muchos especialistas como el inicio del discurso y la práctica del desarrollo a nivel internacional (Riddell, 2007). 
el patrón capitalista. ${ }^{3}$ Los trabajos de Rostein-Rodan (1943) y de Rostow (1956) —dos de los principales teóricos de la modernización- proporcionan un claro ejemplo de lo anterior. Desde su enfoque el desarrollo era entendido en términos puramente económicos y el camino para conseguirlo consistía en avanzar por una serie de etapas mediante recursos materiales - dinero y tecnologías-y valores transferidos del Norte al Sur. ${ }^{4}$ Con el tiempo, y debido a factores diversos, esta visión única sobre el desarrollo fue transformándose. ${ }^{5}$ Es decir, el concepto mismo de desarrollo, sus causas y, consecuentemente, las estrategias para alcanzarlo cambiaron. Así pues, otras teorías y enfoques fueron ganando terreno: la teoría de la dependencia, por ejemplo, sostenía que la explotación de la metrópoli a la periferia era la principal causa del subdesarrollo y argumentaba la necesidad de independizarse de la última (periferia); los modelos de desarrollo centrados en el Estado, por su parte, cuestionaban el patrón capitalista; en ese sentido, el llamado modelo del vuelo del ganso utilizado por Japón y otras economías del Este asiático se mostraba como una importante alternativa; mientras los enfoques en las "necesidades básicas” — surgidos a partir de los trabajos de la Organización Internacional del Trabajo y del Banco Mundial durante la presidencia de Robert McNamarabuscaban dar un rostro más "social" al concepto de desarrollo. ${ }^{6}$ No obstante, a raíz de la crisis del petróleo — primero en 1973 y luego en 1979_, la crisis de la deuda en los países en desarrollo y, sobre todo la llegada de Margaret Thatcher (1979) y Ronald Reagan (1981) al poder en Reino Unido y Estados Unidos, respectivamente, se dio un vuelco en el enfoque sobre el desarrollo hacia un modelo único basado en un conjunto de políticas conocidas como el Consenso de Washington. De ahí que, desde la década de los ochenta y hasta la actualidad el modelo neoliberal se estableció como el paradigma dominante del desarrollo en la escena internacional; así lo demuestra la adopción de esas políticas por las instituciones más relevantes en la materia - Banco Mundial, FMI, OCDE- y un considerable número de países en el mundo.

3. Thiel, 2001.

4. Por Norte nos referimos a los países industrializados. Por otro lado, para más información sobre los primeros teóricos del desarrollo véase Thorbecke, 2000.

5. Se pueden considerar, por ejemplo, factores como el contexto internacional, el descontento en algunos países del Sur hacia las estrategias del Norte, los resultados ambiguos y la propia evolución de la teoría del desarrollo (Willis, 2005).

6. Hodder, 2000; Thiel, 2001; Willis, 2005. 
Más aún, el modelo neoliberal, como cualquier otro, ha sufrido reveses en distintos frentes tales como: el colapso de la economía mexicana y la brasileña en los años noventa, la crisis asiática en $1997,{ }^{7}$ y la crisis económica actual cuyo impacto se ha sentido primordialmente en los países desarrollados. ${ }^{8}$ Adversidades que para muchos han sido, no sin debate, muestra innegable del fracaso de dicho modelo. Fracaso que contrasta con el éxito logrado por economías que, como la china, la japonesa o la coreana, han optado por dejar de lado la ortodoxia neoliberal y han establecido políticas más acordes con sus realidades y necesidades, algo que de acuerdo con José Luis Calva, ha faltado en México:

En México cumplimos casi tres décadas de perseverante sumisión a la ortodoxia del Consenso de Washington, en vez de aplicar una estrategia soberana y pragmática de desarrollo e inserción digna y eficiente en la economía mundial [...] el resultado son casi tres décadas perdidas para el desarrollo y una tremenda regresión en los niveles de ingreso y bienestar de las mayorías nacionales. ${ }^{9}$

Ahora bien, lo importante de lo anterior es reconocer que no existe una receta única para el desarrollo, es decir, una serie de políticas diseñadas "desde afuera" y aplicadas metódicamente mediante las cuales se puedan obtener resultados exitosos en cualquier país. Por el contrario, los casos señalados líneas arriba muestran cómo políticas alternas pueden alcanzar resultados positivos. En esa línea, la situación actual de nuestro país - beneficios concentrados en unos cuantos y no en el país en su conjunto- demanda, como expone José Luis Calva, la búsqueda de dichas alternativas. Más aún, el contexto electoral que se vive en la actualidad presenta una oportunidad inmejorable para plantear una nueva estrategia más acorde con la realidad y con las necesidades de México. Y qué mejor manera de hacerlo que analizando los procesos de éxito en otras economías, no sólo industrializadas, sino también en desarrollo. Lo anterior teniendo presente, como señala Enrique Pino (2012), que si bien "las experiencias concretas de desarrollo no son imitables o reproducibles",

7. Recordando que el éxito de los "tigres asiáticos" era una de las principales cartas de presentación como caso de éxito para los defensores y promotores de dicho modelo (Willis, 2005).

8. Hernández, 2012.

9. Calva, 2012: 17. 
pueden asimilarse de manera creativa y adaptarse "a las circunstancias propias de cada nación". 10

Es precisamente este objetivo el que se persigue en la obra titulada Estrategias económicas exitosas en Asia y América Latina, coordinada por el connotado economista José Luis Calva; segundo volumen - de 18- de la colección "Análisis Estratégico para el Desarrollo" editada de manera conjunta por el Consejo Nacional de Universitarios (CNU) y Juan Pablos Editor. Colección que constituye un ambicioso, pero como vimos antes, necesario esfuerzo de conjuntar, analizar y sobre todo formular "un sistema integral de propuestas viables de políticas públicas" tratando de vincular al sector académico con el gubernamental para encauzar al país en la senda de un "desarrollo sustentable, incluyente, equitativo y democrático". ${ }^{11}$

En este segundo volumen, en el cual participan académicos de diversas instituciones tanto nacionales como extranjeras, se estudian a lo largo de sus 326 páginas los casos exitosos de seis países, más un apartado referente al sistema financiero y su relación con el desarrollo. El libro está dividido en siete secciones: ${ }^{12} 1$. La exitosa estrategia posneoliberal de Argentina. 2. La estrategia económica de Corea del Sur. 3. China: las claves de su crecimiento económico espectacular. 4. Brasil: su estrategia posneoliberal de desarrollo incluyente. 5. India: su camino a la prosperidad. 6. Japón: su salto del subdesarrollo al desarrollo. Y 7. Sistemas financieros y desarrollo: Asia y América Latina. Cada sección, excepto la última, consta de dos artículos que permiten al lector una mayor profundización sobre el tema y la posibilidad de comparar diversas perspectivas en cada proceso, lo que sin duda enriquece el análisis. Por otra parte, la relevancia de Asia, región sobre la que se analizan las estrategias de desarrollo de cuatro de sus economías, no es casualidad, ya que Asia incluye un número importante de historias de éxito en las últimas décadas: el "milagro" japonés, el desarrollo de los tigres —o dragones— asiáticos: Corea del Sur, Singapur, Taiwán y Hong Kong; y el ascenso de China, entre otras que han sido ampliamente documentadas en números anteriores de México y la Cuenca del Pacífico.

10. Pino, 2012: 69.

11. El texto entrecomillado corresponde a los objetivos señalados en la contraportada del libro.

12. Participan académicos de la Universidad Nacional Autónoma de México (UNAM), Universidad de Guadalajara (UdeG), El Colegio de México (Colmex), Universidad Autónoma Metropolitana (UAM), Universidad de Colima (Ucol), Universidad Nacional de Quilmes (UNQ), la Universidad Nacional de San Martín (UNSAM) y el Instituto de Pesquisa Econômica Aplicada de Brasil. 
Retomando la obra, los casos analizados en cada una de las secciones parten de una revisión de los antecedentes y de las causas -internas y externas- que influyeron de manera determinante en la elección de una nueva estrategia de desarrollo en cada país, para después explicar aquellos elementos clave y distintivos que, a decir de los autores, constituyen parte fundamental del éxito alcanzado. ${ }^{13}$ Siguiendo ese orden de ideas, es interesante - y refuerza plenamente el objetivo de la obra- notar que aun cuando las causas que determinan el establecimiento de un nuevo modelo de desarrollo, así como las estrategias, han sido diversas entre los países analizados, el común denominador del éxito gira en torno al pragmatismo y flexibilidad de los modelos utilizados. Modelos en los que tiene lugar una relación sui géneris entre el Estado y el mercado en función de las peculiaridades e intereses de cada país. Aunado a ello, nos invitan a la reflexión sobre las posibles lecciones u oportunidades para México.

Así pues, a partir de la lectura de los capítulos ${ }^{14}$ encontramos que mientras en Argentina la crisis entre 1999 y 2002 —provocada en gran medida por las políticas del Gobierno peronista-neoliberal durante la presidencia de Carlos Saúl Menem (1989-1999) — abrió la puerta para la llegada del kirchnerismo, de la mano de una clase media activa, y con éste de un modelo de capitalismo mixto para hacer frente a las terribles consecuencias sociales y económicas de dicha crisis. ${ }^{15}$ En la República Popular de China (RPC) fueron los magros resultados del modelo de "socialismo real" de la etapa maoísta, combinados con la llegada de Deng Xiaoping al poder del Partido Comunista, los que marcaron el inicio de las reformas económicas y políticas para instaurar un "socialismo de mercado". ${ }^{16}$ Paradójicamente, Argentina pasó de una estrategia basada en el endeudamiento externo que había resultado en la extranjerización de la

13. Éxito que se sostiene a través del uso de diversos indicadores como el incremento del PIB, del comercio internacional, del ingreso real, del empleo o de la productividad sectorial, entre otros.

14. A partir de este punto se trabaja con los textos de Dabat (2012) y de Rofman y Fidel (2012) para el caso de Argentina, y de Gonzáles (2012) y Dussel Peters (2012) en lo concerniente a China.

15. Caída del producto interno bruto y del empleo, disminución del salario real, quiebra de pequeños negocios, un altísimo endeudamiento externo con masivas fugas de capital, crecimiento de la desigualdad y un porcentaje de la población mayor a 50\% por debajo de la línea de la pobreza (Dabat, 2012; Rofman y Fidel, 2012).

16. Durante el periodo maoísta, como señala Gonzáles (2012) se contribuyó "a la disminución del atraso económico", pero "la mayor parte de la población seguía en condiciones de pobreza" (p. 125). 
industria manufacturera, los servicios públicos, y el consecuente achicamiento del aparato y funciones estatales, hacia una en la que se reforzaría el papel del Estado en la economía y se promoverían las exportaciones del sector agrícola generando un vínculo importante entre el sector privado nacional y el Estado, vínculo crucial para el éxito argentino. ${ }^{17} \mathrm{~A}$ la luz de dicha estrategia, el Estado se enfocó en reducir la deuda externa, mantuvo la subvaluación de la moneda para promover las exportaciones - de las que China ha sido uno de los principales compradores-y disminuir las importaciones $\mathrm{y}$, a la vez, aplicó impuestos a las exportaciones de bienes primarios, agropecuarios y extractivos para captar recursos que servirían para fortalecer sus finanzas y vigorizar al sector industrial. Para 2005 el país pagó su deuda con el FMI, lo que aumentó el margen de maniobra al Gobierno sobre las políticas. Así, con la llegada de Cristina Fernández de Kirchner al poder (2007) se enfatizaron las políticas de carácter social, el apoyo a las pequeñas y medianas empresas, e inició la renacionalización de activos de capital de empresas transnacionales.

China, al contrario, comenzó un proceso gradual para establecer un marco normativo que permitiera una mayor participación del sector externo en la economía. Así, el comercio internacional y la inversión extranjera directa (IED) se convirtieron en elementos trascendentales para la transformación de China en una economía industrial. La estrategia china, por tanto, se ha preocupado por generar un ambiente favorable para los agentes económicos, y en disminuir el papel del Estado en la economía. A raíz del ingreso de China a la OMC en 2001, la estrategia se ha redefinido, por un lado, para tratar de adecuarse a los estándares internacionales y, por otro, para concentrarse en el mercado interno - aumentar la demanda interna estimulando el consumo en lugar del ahorro o la inversión - y reducir la dependencia hacia el sector externo, algo entendible en tiempos de crisis económica global. Lo anterior ha dado forma a la nueva política económica con características chinas que basa su éxito en una conjunción de políticas de apertura económica y de corte poskeynesiano - política fiscal expansiva y monetaria proactiva-. Por lo anterior, parece que mientras un país busca alejarse de las reglas impuestas por los organismos internacionales, el otro se acerca, pero ambos conscientes de la necesidad de que el Estado participe, de una u otra manera, en la actividad

17. Dabat, 2012. Por su parte, el artículo de Rofman y Fidel (2012) ofrece una excelente tabla comparativa para el análisis en profundidad de las implicaciones del cambio de modelo, o mejor aún, de cada uno de los modelos en disputa. 
económica. En este punto es importante señalar, como hacen los autores, que aunque las estrategias han obtenido resultados positivos - Argentina creciendo entre 2003 y 2008 a una tasa del orden de $9 \%^{18}$ y China con un crecimiento anual promedio de su PIB real por encima de $9 \%$ entre 1979 y 2007-, ${ }^{19}$ los retos que enfrentan están a la orden del día. ${ }^{20}$ Más aún, podría ser prematuro considerar el caso argentino como uno de éxito similar al de las economías asiáticas; aunque se debe reconocer que, pese al poco tiempo de implementación, brinda pistas sobre posibles alternativas para México, por ejemplo en cuanto a la serie de programas utilizados por el país sudamericano para atenuar los efectos de la presente crisis económica global. Los posibles escenarios futuros de la economía argentina se tratan en el segundo capítulo de la primera sección.

Por último, en torno a China, mención aparte merece el breve artículo de Enrique Dussel Peters que aborda el tema de la relación bilateral entre MéxicoChina y plantea, con razón, la necesidad de realizar esfuerzos institucionales para fortalecer dicha comunión. La importancia de ello radica en el hecho de que China es el segundo socio comercial en importancia para nuestro país y uno de los principales inversionistas entre los países en desarrollo, pero México no ha podido aprovechar las oportunidades que esto podría representar, como sí lo han hecho otros países latinoamericanos. Dussel destaca el esfuerzo realizado, en ese sentido, por el Grupo de Trabajo México-China que se reunió entre 2008-2010 con la finalidad de lograr una agenda estratégica para el diálogo y el acercamiento entre ambos países en torno a tres ejes prioritarios, a saber: instituciones binacionales; migración y turismo; y economía, comercio, inversión e infraestructura.

Contrario a los procesos señalados líneas arriba, los casos de Japón y Corea del Sur parecen más próximos entre sí. ${ }^{21}$ Resaltan, en cuatro aspectos, las semejanzas entre los "modelos" de ambos países, aunque es importante mencionar que el estudio de los artículos del libro permite también identificar las diferencias sustanciales.

18. Rofman y Fidel, 2012.

19. Yeoh y Loh, 2008.

20. Véanse las secciones 1 y 3 del texto reseñado para profundizar en dichos retos.

21. La sección referente a Corea del Sur y Japón se basa en los textos de Pino (2012), Estrada y Landa (2012), Falck (2012) y Román (2012). Véanse la segunda y tercera sección de la obra. 
En primer lugar, ambas economías, si bien desde posiciones desiguales ${ }^{22}$ iniciaron su "milagro económico" enfrentando un contexto internacional marcado por la Guerra Fría en el que el apoyo estadounidense, a partir de su cruzada anticomunista, les benefició de manera significativa. ${ }^{23}$

En segundo lugar, el éxito económico de uno y otro descansó, en gran medida, en el papel del Estado como facilitador y guía del desarrollo, pues a través de sus políticas protegió a las empresas de la competencia externa y las promovió con subsidios, incentivos a la exportación y la política monetaria, suministrando insumos y otras medidas, además de apoyar a los sectores estratégicos para la industrialización en cada caso. El uso de políticas selectivas generó un estrecho vínculo entre el sector público y el sector privado privilegiado. De ahí que tanto el triángulo de hierro en Japón -Partido Demócrata Liberal, burocracia y los grandes grupos industriales (Keiretsu)—, como el pacto entre el Gobierno y los grandes conglomerados (Chaebols) en Corea del Sur se constituyeran como un rasgo característico de los "modelos" y una asociación favorable para el desarrollo.

La preferencia en utilizar inversión y ahorro interno para financiar la estrategia de desarrollo, disminuyendo así la dependencia hacia el exterior y permitiendo una mayor agencia, sería un tercer punto en común. Más aún, aunque a principios de la década de los noventa ambos países modificaron el régimen de la inversión extranjera directa (IED), la preponderancia del ahorro interno se ha mantenido, especialmente en el caso de Japón.

En cuarto lugar es necesario señalar una serie de elementos que, a decir de los autores, fueron igualmente determinantes que los anteriores para elevar la calidad de vida en ambos países y que pueden ser especialmente relevantes para México: 1 . El diseño de un plan a largo plazo adecuado a la realidad del país; tanto Corea del Sur como Japón iniciaron su proceso de industrialización atendiendo al principio de la dotación de factores y no de

22. Japón ya había experimentado una fase previa de industrialización entre 1868 y 1940 en la que el uso de los excedentes del sector agrario - oferta de mano de obra y fuente de divisassirvieron como insumos para el desarrollo industrial pero, como se sabe, la capitulación de la Segunda Guerra Mundial fue catastrófica para el país. Y Corea daba sus primeros pasos hacia la industrialización.

23. Por un lado, Corea del Sur recibió una importante transferencia de recursos económicos que en un primer momento impulsaron el proceso de industrialización y, por otro, Japón pudo concentrar sus esfuerzos y su presupuesto en la reconstrucción y el crecimiento económico, ya que Estados Unidos se ocupó de los asuntos relacionados con la seguridad (Pino, 2012; Román, 2012). 
acuerdo con recetas externas. 2. La definición de un interés común entre los principales actores. 3. La importancia de una burocracia capaz de "guiar administrativamente" el proceso de desarrollo en torno al objetivo común -este punto, más relacionado con Japón, es claramente expuesto en el texto de Román (2012), en el que se acentúan los elementos culturales que han dado forma a la burocracia japonesa, su relación con otras élites y su tarea para lograr el crecimiento económico. 4. La capacidad institucional para responder a las crisis y a los retos que se van presentado; los textos dan prueba de ello, mostrando a su vez la importancia del uso de políticas públicas flexibles y pragmáticas. 5. La preocupación constante en mejorar el nivel educativo y la calidad de la mano de obra, lo que permitió la asimilación de tecnologías extranjeras, la posibilidad de desarrollar nuevas tecnologías y transformar la estructura económica, y el aumento de la productividad; relacionado con este último punto, la estrategia utilizada por Corea del Sur para incrementar la productividad es analizada en el texto de Estrada y Landa (2012); asimismo los autores exponen las notorias diferencias entre Corea del Sur y México respecto a la inversión en investigación y desarrollo (I+D). Los datos refuerzan el argumento a favor de una mayor inversión en la educación presente en la mayoría de los artículos del libro. ${ }^{24} \mathrm{Y}$ 6. No descuidar el sector de las pymes sino coadyuvarlas, como se hizo en Japón, para vincularlo con las cadenas productivas del sector exportador.

India y Brasil completan los procesos explorados en el libro. ${ }^{25} \mathrm{Al}$ igual que China, ambos son parte de los llamados BRIC, pero el interés en el estudio de sus "modelos" económicos actuales en México es relativamente más reciente. La sección dedicada a la India describe brevemente los puntos de quiebre y las principales características de su estrategia de desarrollo. En ese sentido, y de acuerdo con los autores, para entender el "modelo" de desarrollo hindú primeramente debemos advertir el apegó del país, tras su independencia, a los ideales del crecimiento económico planificado — derivado de la experiencia de la Unión Soviética - y de la democracia. Ideales que se ven plasmados en

24. Corea del Sur, por ejemplo, en 2008 invirtió alrededor de $3.13 \%$ del PIB en dicho ramo, contribuyó con $4.18 \%$ del total de las patentes registradas en el mundo y contó con 9.7 investigadores por cada 1,000 integrantes de la PEA; por su parte, México en ese mismo año destinó sólo $0.37 \%$ del PIB, generó $0.03 \%$ del total de las patentes y contó con 0.86 investigadores por cada 1,000 habitantes parte de la PEA (Estrada y Landa, 2012).

25. La sección se basa en los textos de Bizberg (2012), De Castro (2012), Preciado (2012) y Carballido (2012). Véanse la cuarta y quinta sección de la obra. 
el establecimiento de la Comisión Nacional de Desarrollo como órgano rector del proceso de industrialización y en el que participan el primer ministro, ministros y funcionarios del gobierno federal y estatal, investigadores y representantes del sector privado quienes, en conjunto, diseñan la estrategia a largo plazo e identifican las prioridades del país. Así, bajo el liderazgo de Jawaharlal Nehru y de una élite educada, dio inicio la primera etapa de desarrollo (1947-1980) con el objetivo de lograr la autosuficiencia, una rápida industrialización mediante la sustitución de importaciones, con énfasis en el capital nacional y de carácter igualitario. El Estado, además de planificar, participaba como inversionista y regulaba el mercado.

Posteriormente, en 1980 se aplicaron una serie de reformas con el objetivo de incrementar la productividad: se liberalizó el régimen de las importaciones y se abrió la puerta al capital extranjero, se introdujeron reformas fiscales para reducir el déficit del Gobierno, se incentivó la inversión privada que en la actualidad es mayoritaria, pero se mantuvieron regulaciones en algunos sectores. Asimismo, se robusteció la capacitación de los trabajadores que, combinado con una mano de obra barata, ha sido uno de los factores que ha convertido a India en un beneficiario de la deslocalización de los procesos de producción de empresas, mayormente estadounidenses. Finalmente, a pesar de que entre 1947 y 2009 en India salieron de la pobreza aproximadamente 300 millones de personas, hay importantes problemas por combatir tales como la poca importancia relativa del sector manufacturero, la desigualdad económica y de género, la falta de infraestructura, la cuestión alimentaria, la educación universal, entre otros. No obstante, el ejemplo de India refuerza, como señala Preciado (2012), lo importante de la planeación a mediano y largo plazos y del desarrollo de una estrategia endógena.

Por su parte, la sección de Brasil ofrece dos artículos que en conjunto brindan un panorama completo de lo que ha venido ocurriendo en el país. El primero, de Ilán Biezberg (2012), lo hace a través de un análisis comparativo con México en el que busca demostrar cómo es que las coaliciones sociopolíticas - relaciones entre el Estado, las organizaciones obreras y las empresariales - han conformado un subtipo de capitalismo en cada país: uno guiado por el mercado interno y con fuerte intervención estatal en Brasil, y otro basado en la subcontratación internacional con poca intervención estatal en México. Análisis que a su vez procura una interesante perspectiva para analizar la situación actual en México. El segundo, de Jorge Abrahâo De Castro (2012), ahonda en el "modelo" brasileño desde uno de los que muchos 
consideran su aspecto más exitoso: la política social. El autor profundiza su análisis definiendo el concepto y explicitando los objetivos que ésta persigue, mostrando el andamiaje institucional y organizacional que se ha formado en torno a la misma, y evaluando sus resultados en torno a la población y a la economía nacional.

La sustitución de importaciones trazó el camino hacia la industrialización tanto en México como en Brasil. Sin embargo, este modelo surgió y funcionó en el entorno de estructuras sociopolíticas muy diferentes. Así, en Brasil fueron el sector agrícola e industrial los que fomentaron la industrialización con un Estado mediador de los intereses entre ambos. Marco en el cual la industrialización era una herramienta para la modernización y la unificación nacional. En cambio, en México la industrialización fue guiada por el Estado con el objetivo de fortalecer al régimen político cooptando a otros sectores mediante los subsidios, la protección estatal y las inversiones directas. El Estado se convirtió en instrumento del régimen cuya estabilidad era el fin superior; cabe apuntar que la legitimidad del partido en el poder estaba en función de su herencia revolucionaria y en el mantenimiento de los beneficios a los sectores protegidos. Lo anterior trazó una trayectoria histórica disímil entre ambos países. Tendencia que se amplio más a raíz de las crisis económicas que impactaron al continente, en especial la crisis de la deuda en los setenta. Brasil se vio forzado a utilizar sus propios recursos, inversión estatal e incentivo del mercado interno para continuar su modernización reduciendo su dependencia del exterior. Mientras que México aprovechó el descubrimiento de "vastas reservas petroleras" y se convirtió en un importante exportador. Este "modelo", con una sujeción considerable a las exportaciones de petróleo, mostró sus límites a inicio de los ochenta, "obligando" al país a recurrir a recursos del FMI, lo que implicó el inicio de las reformas estructurales. Los caminos recorridos por uno y otro, y las coaliciones que se formaron en uno y otro, resultaron en políticas económicas, laborales y sociales mucho más complementarias en el caso de Brasil que en el de México.

Las políticas sociales en Brasil, como se apuntó, han sido potentes. Éstas han ponderado dos cuestiones: la protección social y la promoción social. Ambas se han perseguido mediante una serie de políticas sectoriales y transversales, planificadas a largo plazo, impulsadas con recursos públicos. ${ }^{26}$ Lo anterior

26. Por políticas transversales se hace referencia a aquéllas destinadas a la igualdad de género y racial, entre niños, adolescentes y ancianos, entre otras (De Castro, 2012). 
ha requerido la construcción de un considerable andamiaje institucional en los tres niveles de gobierno, e incluso con participación del sector privado, en cada uno de los sectores sociales como la salud, la educación, la asistencia social, la vivienda, entre otros. De este aparato institucional surge una plétora de beneficios y programas ofrecidos diariamente a millones de ciudadanos. Ahora bien, a pesar de los retos de coordinación que aún existen, el avance en la calidad de vida es notorio al revisar los datos. Más aún, el texto de De Castro muestra que se han identificado las políticas que tienen repercusiones positivas - efectos multiplicadores - en el PIB y en el ingreso real de las familias, así como las que contribuyen significativamente para disminuir la desigualdad en el ingreso al interior del país. En virtud de ello es importante profundizar en su estudio y buscar aquellas que pudieran ser adaptadas a las circunstancias de México.

El último capítulo de la obra, presentado por Geneviève Marchini (2012), analiza el impacto que el desarrollo del sistema financiero doméstico ha tenido en el crecimiento económico de algunas naciones del este y sudeste asiático, y de Latinoamérica. El estudio de esta relación, explica la autora, ha sido muchas veces ignorado tanto en el campo de la teoría económica como por las teorías del desarrollo. Más aún, es hasta la década de los setenta, con el surgimiento del enfoque financiero, que se inicia el debate. ${ }^{27}$ Debate que se ha ido profundizando debido a la acumulación de evidencias empíricas, es decir, de los resultados obtenidos por las naciones al aplicar distintas políticas. Al respecto Marchini sostiene, primero, que la esfera financiera puede contribuir al crecimiento económico facilitando "el financiamiento de la actividad productiva, de la inversión y de la innovación”, y que a su vez el crecimiento económico generará "el desarrollo de los intermediarios financieros"; y segundo que las políticas de liberalización financiera, por sí solas y debido a la existencia de las fallas de mercado, no establecen las condiciones necesarias para el desarrollo de un sistema financiero "apto para financiar [...] la economía real" de determinado país. ${ }^{28}$

En virtud de lo anterior, subraya la autora, el análisis de los sistemas financieros internos de economías en desarrollo con altas - y bajas - tasas

27. Los postulados centrales del enfoque financiero son: a) el desarrollo financiero acelera el crecimiento económico; b) las políticas financieras fomentan o inhiben el desarrollo financiero, y c) hay una relación causal entre liberalización financiera, desarrollo y crecimiento económico (Marchini, 2012: 302).

28. Marchini, 2012: 305. 
de crecimiento económico, permite hacer recomendaciones valiosas para otras economías como la mexicana. Precisamente este análisis, que abarca el periodo de 1960 hasta 2009, es uno de los objetivos del texto. Partiendo de sus resultados, se señala que México muestra niveles de desarrollo financiero muy bajos ("subdesarrollo financiero") en relación con las economías asiáticas revisadas. En éstas el sistema financiero proporciona grandes volúmenes de crédito al sector privado y los mercados de valores - con un mayor número de empresas o un mayor tamaño en relación con el PIB- tienen un papel mucho más relevante en cuanto a la inversión. Por otro lado, la exploración de los casos también expone que una de las causas del "subdesarrollo financiero" en América Latina radica en la manera en que dichas economías se han incorporado en el sistema financiero internacional. Buena parte de los países latinoamericanos examinados, incluido México, lo han hecho mediante la aplicación de políticas neoliberales ortodoxas. En contraparte, las naciones asiáticas consideradas lo han hecho a través de políticas pragmáticas y de forma gradual. Ambos elementos parecen reforzar sus planteamientos iniciales. El desarrollo, o subdesarrollo, del sistema financiero doméstico impacta en el crecimiento económico y las políticas pragmáticas han sido más favorables para desarrollar dicho sistema.

Para concluir, se puede decir que en lo general la obra cumple con el objetivo de brindar una revisión cabal de algunas de las más exitosas estrategias de desarrollo en los últimos años. La mayoría de los textos acentúan aquellos elementos y políticas que no solamente han contribuido al éxito de cada uno de los países analizados, sino que pudieran se ejemplo para el caso de México. En ese sentido, los textos de Dabat, Pino, Gonzáles, Marchini y las secciones dedicadas a Brasil y Japón son especialmente enriquecedores. Políticas pragmáticas adecuadas a la "realidad" nacional constituyen un común denominador en los artículos, así como una mayor, pero eficiente, participación estatal en determinados ámbitos de la actividad económica. Así pues, este volumen sobre Las estrategias económicas exitosas en Asia y en América Latina es tan recomendable para el público en general, como para estudiosos del tema y colaboradores en el sector gubernamental. ๓ฺy 


\section{Referencias bibliográficas}

Bizberg, Ilán (2012), "Brasil y México: Dos tipos de capitalismo", en Calva, José Luis (coord.), Estrategias económicas exitosas en Asia y en América Latina, México: Consejo Nacional de Universitarios/Juan Pablos Editores.

Calva, José Luis (2012), "Prólogo", en Calva, José Luis (coord.), Estrategias económicas exitosas en Asia y en América Latina, México: Consejo Nacional de Universitarios/Juan Pablos Editores.

Carballido, Laura (2012), "El crecimiento económico de India (1991-2011)", en Calva, José Luis (coord.), Estrategias económicas exitosas en Asia y en América Latina, México: Consejo Nacional de Universitarios/Juan Pablos Editores.

Dabat, Alejandro (2012), "El nuevo rumbo de la economía argentina bajo el kirchnerismo" en Calva, José Luis (coord.), Estrategias económicas exitosas en Asia y en América Latina, México: Consejo Nacional de Universitarios/ Juan Pablos Editores.

De Castro, Jorge Abrahâo (2012), "Política social en el Brasil contemporáneo", en Calva, José Luis (coord.), Estrategias económicas exitosas en Asia y en América Latina, México: Consejo Nacional de Universitarios/Juan Pablos Editores.

Estrada, José Luis, y Heri Óscar Landa (2012), “El crecimiento económico de Corea del Sur" en Calva, José Luis (coord.), Estrategias económicas exitosas en Asia y en América Latina, México: Consejo Nacional de Universitarios/ Juan Pablos Editores.

Hernández, Roberto (2010), "La economía de China en el contexto de la crisis económica mundial 2007-2009”, México y la Cuenca del Pacífico, vol. 13, núm. 39, septiembre-diciembre, Guadalajara: Universidad de Guadalajara, pp. 47-70.

Hodder, Rupert (2000), Development Geography, Florencia: Routledge.

Marchini, Geneviève (2012), "Liberalización, desarrollo e integración financiera en las economías asiáticas: Una perspectiva comparada con México y América Latina", en Calva, José Luis (coord.), Estrategias económicas exitosas en Asia y en América Latina, México: Consejo Nacional de Universitarios/Juan Pablos Editores.

Pino, Enrique (2012), "Corea del Sur: Lecciones de una estrategia alternativa de desarrollo", en Calva, José Luis (coord.), Estrategias económicas exitosas 
en Asia y en América Latina, México: Consejo Nacional de Universitarios/ Juan Pablos Editores.

Preciado, Benjamín (2012), "El triunfo de la planeación económica. La experiencia india durante sesenta años", en Calva, José Luis (coord.), Estrategias económicas exitosas en Asia y en América Latina, México: Consejo Nacional de Universitarios/Juan Pablos Editores.

Riddell, Roger C. (2007), Does Foreign aid Really Works, Oxford: Oxford University Press.

Rofman, Alejandro, y Carlos Fidel (2012), "Modelos económico-sociales en Argentina: La disputa continúa”, en Calva, José Luis (coord.), Estrategias económicas exitosas en Asia y en América Latina, México: Consejo Nacional de Universitarios/Juan Pablos Editores.

Román, Alfredo (2012), "El papel de la burocracia en la estrategia de desarrollo económico de Japón”, en Calva, José Luis (coord.), Estrategias económicas exitosas en Asia y América Latina, México: Consejo Nacional de Universitarios/Juan Pablos Editores.

Thiel, Reinold E. (2001), Teorías del desarrollo: Nuevos enfoques y problemas, Caracas: Nueva Sociedad.

Thorbecke, Erik (2000), "The evolution of the development doctrine and the role of foreign aid, 1950-2000", en Tarp, Finn (ed.), Foreign Aid and Development: Lessons Learned and Directions for the Future, Florencia: Routledge. Willis, Katie (2005), Theories and Practices of Development, Londres/Nueva York: Routledge.

Yeoh, Emile, y Joanne Loh (2008), China in the World: Contemporary Issues and Perspectives, Kuala Lumpur: University of Malaya. 


\section{Historia de las relaciones \\ internacionales de México \\ 1821-2010}

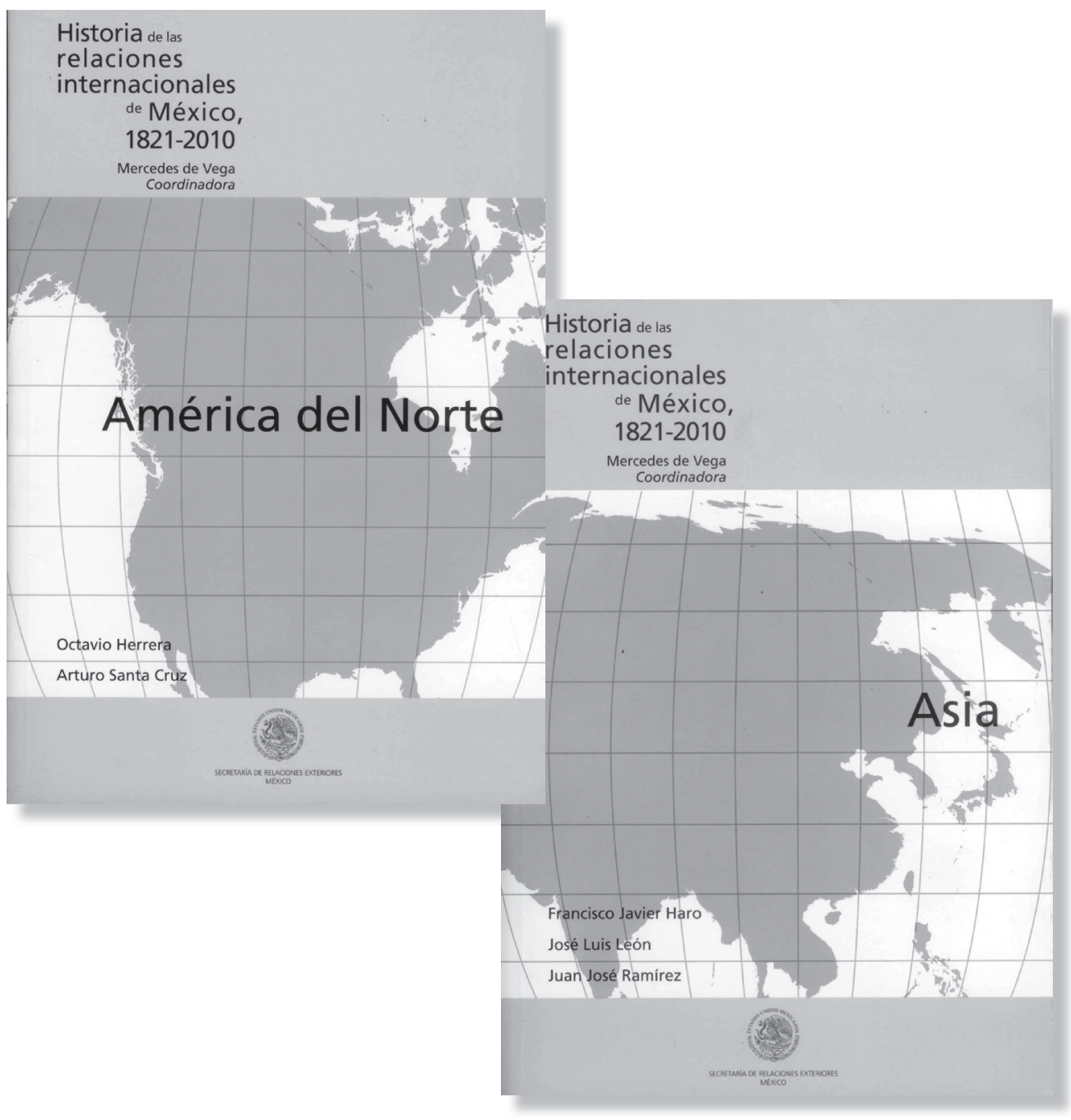

